\title{
Vertical Transmission of SARS-CoV-2 to Newborns in COVID-19 Infected Mothers: A Cross-sectional Study
}

\author{
Hemraj Narkhede ${ }^{1} \odot$, Mahesh R Asalkar ${ }^{2}$, Surkyant Munload ${ }^{3}$, Vipulachandra $Y^{4}$, Tushar V Patil ${ }^{5}$
}

\begin{abstract}
Aim and objective: To establish the presence or absence of vertical transmission of SARS-CoV-2 in patients who delivered during the study period. Study design: Cross-sectional study was conducted from May 2020 to October 2020 at dedicated COVID hospital. During the study period, all SARS-CoV-2-positive patients who delivered at the study institution were studied to find SARS-CoV-2 infection in newborn by intrauterine transmission. Newborns were screened within 60 minutes of delivery for vertical transmission of SARS-CoV-2 infection by nasopharyngeal swab test for RT-PCR.

Results: Two hundred one COVID positive pregnant women enrolled in the study. Two newborns tested SARS-CoV-2 positive by RT-PCR test: One was delivered by vaginal route and other by caesarian section. Both babies who were tested SARS-CoV-2 positive by RT-PCR test were born after EDD (>40 weeks) to low-risk mothers. Time interval between maternal test positivity and delivery interval was 7-14 days.

Conclusion: The rate of VT is $0.99 \%$ in our study. The possibility of vertical transmission is more in postdated pregnancy. There was no relation seen in our study with the severity of disease or symptoms of infection at the time of admission and vertical transmission, but it needs to be further studied. We did not notice the association of high-risk obstetrics conditions and vertical transmission.

Keywords: Coronavirus, COVID-19, Intrauterine transmission, Perinatal, Pregnancy, SARS-CoV-2, Vertical transmission.

Journal of South Asian Federation of Obstetrics and Gynaecology (2021): 10.5005/jp-journals-10006-1917
\end{abstract}

\section{INTRODUCTION}

Vertical transmission (VT) of acute severe respiratory syndrome coronavirus 2 (SARS-CoV-2) is of great concern for the management of infected antenatal patients. Systematic reviews with sample size ranging from 11 to 114 concluded that there is no vertical transmission of COVID-19. ${ }^{1-4}$ With regard to vertical transmission, there is emerging evidence to suggest that vertical transmission is possible; ${ }^{5}$ however, there are serious limitations to the available evidence. ${ }^{6}$ COVID-19 virus is detected in amniotic fluid, suggesting possible transplacental or transamniotic membrane transfer of infection to newborn. ${ }^{7}$ RT-PCR at birth should be able to detect the former mode of transmission.

Although multiple infectious vectors have been shown to be capable of vertical transmission, the possibility of vertical transmission of SARS-CoV-2 from the infected mother to the fetus or neonate has been a point of a recent debate. This study is an endeavor to find out the presence or absence of vertical transmission of SARS-CoV-2 in antenatal women.

\section{Materials and Methods}

Cross-sectional study was conducted from May 2020 to October 2020 at Postgraduate Institute and YCM Hospital Pimpri, Pune (Maharashtra), which was declared as dedicated COVID hospital during the COVID pandemic. During the study period, all SARS-CoV2-positive (by RT-PCR/rapid antigen test/lgM antibodies) patients who delivered at the study institution were enrolled for the study.

\section{Aims}

To establish the presence or absence of vertical transmission of SARS-CoV-2 among newborns delivered to mothers having antenatal SARS-CoV-2 infection.
${ }^{1,2,4}$ Department of Obstetrics and Gynecology, PCMC'S PGI-YCM Hospital, Pimpri, Maharashtra, India

${ }^{3}$ Department of Pediatrics, PCMC'S PGI-YCM Hospital, Pimpri, Maharashtra, India

${ }^{5}$ Department of Pathology, PCMC'S PGI-YCM Hospital, Pimpri, Maharashtra, India

Corresponding Author: Hemraj Narkhede, Department of Obstetrics and Gynecology, PCMC'S PGI-YCM Hospital, Pimpri, Maharashtra, India, Phone: +91 8097977917, e-mail: hemrajrn@gmail.com

How to cite this article: Narkhede $H$, Asalkar MR, Munload S, et al. Vertical Transmission of SARS-CoV-2 to Newborns in COVID-19 Infected Mothers: A Cross-sectional Study. J South Asian Feder Obst Gynae 2021;13(4):230-235.

Source of support: Nil

Conflict of interest: None

\section{Objectives}

Primary: To utilize RT-PCR test in newborn for the diagnosis of vertical transmission

Secondary: (1) To study the clinical profile of SARS-CoV-2-positive patients who delivered during the study period. (2) To find out vertical transmission rate among the SARS-CoV-2-positive deliveries if VT is present.

\section{Inclusion Criteria}

- All antenatal women delivered during the study period at our center who were diagnosed to have SARS-CoV-2 infection by appropriate test within the last 28 days of delivery.

() The Author(s). 2021 Open Access This article is distributed under the terms of the Creative Commons Attribution 4.0 International License (https://creativecommons. org/licenses/by-nc/4.0/), which permits unrestricted use, distribution, and non-commercial reproduction in any medium, provided you give appropriate credit to the original author(s) and the source, provide a link to the Creative Commons license, and indicate if changes were made. The Creative Commons Public Domain Dedication waiver (http://creativecommons.org/publicdomain/zero/1.0/) applies to the data made available in this article, unless otherwise stated. 
- Gestational age more than 28 weeks at the time of delivery is included in the study.

\section{Exclusion Criteria}

- Patients delivered in emergency as suspect for SARS-CoV-2; however, reports of the patient (positive/negative) came after delivery are excluded from the study.

- Refusal of consent for participation in the study.

- Gestational age less than 28 weeks

\section{Technique/Methodology}

We conducted a prospective, single-center, cross-sectional study at PCMC'S PGI and YCM Hospital, Pune (tertiary teaching hospital), which was dedicated COVID hospital from May 2020 to October 2020. Sample size was all pregnant women diagnosed to have SARS-CoV-2 infection with gestational age more than 28 weeks admitted and delivered during the study period of 6 months. An informed consent of the admitted pregnant women was taken for participation in the study. Data were collected in standard format, which included history, demographic profile, and investigations such as complete blood count, coagulation profile, serum ferritin, C-reactive protein, neutrophil lymphocyte ratio, renal function test, and liver function test.

Nasopharyngeal swab of newborn is collected, irrespective of status of newborn like live/fresh stillbirth/macerated stillbirth. Screening protocol for newborn is described in Flowchart 1 and Table 1.

Newborns were daily assessed for signs of SARS-CoV-2 infection till discharge. Postnatal maternal management was continued as per ICMR guidelines and neonatal postnatal management was as per MOHFW and ICMR guidelines.

\section{Ethical Aspect}

Institutional Ethics Committee approval was obtained and confidentiality of the data maintained throughout the study.

\section{Statistical Analysis}

Statistical analysis was done using Epi Info7 software.

\section{Data Collection}

Data were collected in uniform, consistent, and reliable manner by trained qualified doctors with the use of standard proforma. Key variables such as age, parity, duration of gestation, physical examination, results of routine laboratory and profile for COVID-19, mode of delivery, antepartum high risk, neonatal RT-PCR swab reports and complications and intrapartum events were recorded.

\section{Results}

Two hundred one COVID positive pregnant women enrolled in the study who fulfilled inclusion criteria. We had 64 pregnant women admitted as suspect for SARS-CoV-2 at the time of admission; however, SARS-CoV-2 test results were not available till delivery; these patients are excluded from the study, irrespective of their test result due to strict inclusion criteria of SARS-CoV-2 test positive before labor, for enrollment. Total SARS-CoV-2-positive pregnant patient treated at our center is 265, out of which 201 fulfilled inclusion criteria. All these 201 SARS-CoV-2-positive women delivered 201 newborns. These newborns were screened for vertical transmission of SARS-CoV-2 by nasopharyngeal swab test by RT-PCR for SARS-CoV-2 (Table 1).

Table 2 shows that most patients were in the age-group of 25-30 (40.2\%), followed by $21-24$ (34.8\%). Most patients were from lower socioeconomic class, 110 (55\%). Ninety-four (46.7\%) patients were para 1, followed by 77 (38.3\%) nullipara and 30 (14.9\%) were multipara. Out of 201 enrolled antenatal patients, 172 (85\%) women detected SARS-CoV-2 positive by RT-PCR, 27 (13\%) by rapid antigen test, and $2(0.99 \%)$ by IgM (Table 2$)$. Most patients were asymptomatic (Category A) 93.1\%, while we did not come across severe infection requiring ventilator/BiPAP, etc., in our study.

Table 3 shows that out of 201 patients, 113 (56.2\%) underwent LSCS and 85 (42.2\%) were FTND, while 3 (1.4\%) had instrumental delivery. Out of two newborns who tested SARS-CoV-2 positive by RT-PCR test, one was delivered by vaginal route and other by caesarean section. Preterm deliveries $<37$ weeks were 26 (12.9\%), while rest were term deliveries 37 weeks onward. Both babies who were tested SARS-CoV-2 positive by RT-PCR test were born after $\operatorname{EDD}(>40$ weeks).

Overall, two newborns were tested SARS-CoV-2 positive by RT-PCR after birth, which showed the possibility of vertical transmission of SARS-CoV-2 and vertical transmission rate in our study was 0.99 (\%)

Obstetric high risks and other comorbidities in the mothers were noted. One hundred forty-eight mothers did not have any high-risk or comorbid conditions, while 53 patients showed highrisk factors as mentioned in Table 4. Both babies who were tested positive for RT-PCR were born to low-risk mothers. Our study also had 10 cases of premature rupture of membrane; however, none of the cases showed vertical transmission. For both babies who were positive for SARS-CoV-2 RT-PCR test, time interval between maternal test positivity and delivery was 7-14 days (Table 5), while all babies delivered before 7 days of maternal test for SARS-CoV-2 positivity had negative test for RT-PCR.

Flowchart 1: Flow diagram of study participants

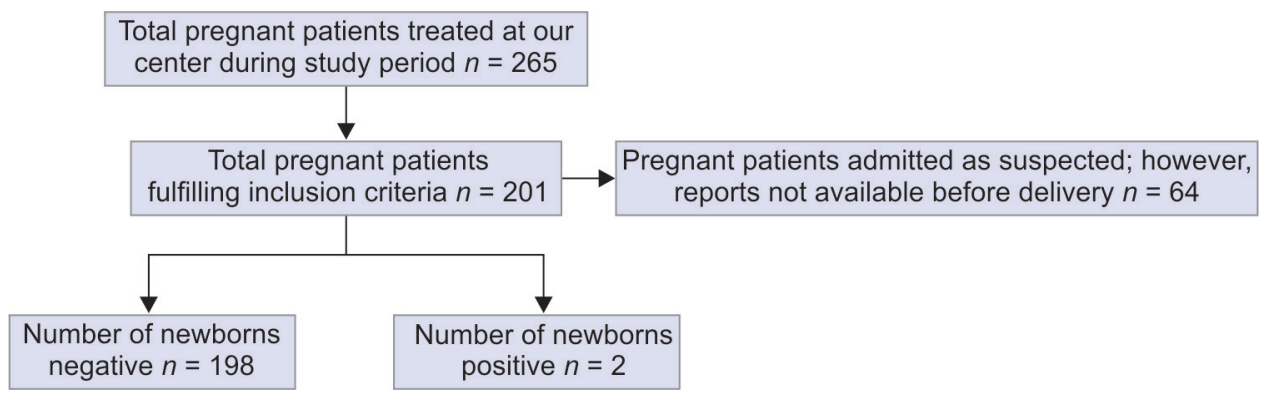


Table 1: Neonatal testing protocol

\begin{tabular}{ll}
\hline Place & In operation theatre/labor room \\
\hline Site & Newborn nasopharynx \\
Time & Within 60 minutes of delivery \\
Collection & $\begin{array}{l}\text { By pediatric resident doctor wearing full PPE and } \\
\text { trained in swab collection }\end{array}$ \\
\hline
\end{tabular}

Table 2: Demographic and clinical profile of COVID-19 positive mothers

\begin{tabular}{|c|c|c|}
\hline & Number & Percentage \\
\hline \multicolumn{3}{|l|}{ Age-group } \\
\hline$<20$ & 16 & 7.9 \\
\hline $21-24$ & 70 & 34.8 \\
\hline $25-30$ & 81 & 40.2 \\
\hline $31-35$ & 26 & 12.9 \\
\hline$>36$ & 8 & 3.9 \\
\hline \multicolumn{3}{|c|}{ Socioeconomic status } \\
\hline High & 27 & 13.4 \\
\hline Middle & 64 & 31.8 \\
\hline Lower & 110 & 55.7 \\
\hline \multicolumn{3}{|l|}{ Parity score } \\
\hline 0 & 77 & 38.3 \\
\hline 1 & 94 & 46.7 \\
\hline 2 or more & 30 & 14.9 \\
\hline \multicolumn{3}{|c|}{ Test used for diagnosis of maternal infection } \\
\hline RT-PCR & 172 & 85.5 \\
\hline Rapid antigen & 27 & 13.4 \\
\hline $\lg \mathrm{M}$ & 2 & 9.9 \\
\hline \multicolumn{3}{|c|}{ COVID severity in mothers as per ICMR classification } \\
\hline Asymptomatic & 187 & 93.1 \\
\hline Mild & 14 & 6.9 \\
\hline Moderate & 0 & 0 \\
\hline Severe & 0 & 0 \\
\hline
\end{tabular}

Clinical management protocol: COVID-19 Government of India Ministry of Health and Family Welfare Directorate General of Health Services (EMR Division) Version 5 03.07.20

Table 3: Obstetric outcome and neonatal COVID test results

\begin{tabular}{lcc}
\hline Mode of delivery $(N=201)$ & & Newborn positive \\
\hline LSCS & $113^{*}(56.2 \%)$ & 1 \\
FTND & $85^{*}(42.2 \%)$ & 1 \\
Instrumental & $3(1.4 \%)$ & 0
\end{tabular}

Gestational age at the time of delivery

$(N=201)$

$\begin{array}{lcl}28-34.6 \text { weeks } & 6(2.9 \%) & 0 \\ 35-36.6 \text { weeks } & 20(9.9 \%) & 0 \\ 37-39.6 \text { weeks } & 157(78.1 \%) & 0 \\ >40 \text { weeks } & 18^{* *}(8.9 \%) & 2\end{array}$

No. of newborns screened and RT-PCR positive test results Newborns screened RT-PCR positive Overall RT-PCR 2012 positivity rate $0.99 \%$

*Out of two newborns tested positive by RT-PCR test, one was delivered by LSCS and other had full-term normal delivery; ${ }^{* *}$ Two newborns tested positive by RT-PCR test were delivered after EDD (G. age $>40$ weeks)
Table 4: Associated obstetric high-risk condition

\begin{tabular}{lccc}
\hline & & \multicolumn{2}{c}{ Newborn test } \\
\cline { 3 - 4 } Condition & Number & Negative & Positive \\
\hline Preeclampsia & 7 & 7 & 0 \\
Abruptio placenta & 2 & 2 & 0 \\
Diabetes/gestational diabetes & 1 & 1 & 0 \\
PROM/PPROM & 10 & 10 & 0 \\
Previous LSCS & 14 & 14 & 0 \\
Oligohydramnios & 5 & 5 & 0 \\
Anemia & 14 & 14 & 0 \\
No high risks/comorbidities & 148 & 146 & 2 \\
\hline$N \geq 201$ as one patient can have & more than & one comorbidity/ \\
high-risk condition & & &
\end{tabular}

Table 5: Date of test mother and time of delivery ${ }^{*}$

\begin{tabular}{lccc}
\hline & & \multicolumn{2}{c}{ Newborn test } \\
\cline { 3 - 4 } Days from test result & Number of patients & Negative & Positive \\
\hline 1 & 35 & 35 & 0 \\
2 & 13 & 13 & 0 \\
3 & 18 & 18 & 0 \\
4 & 15 & 15 & 0 \\
5 & 12 & 12 & 0 \\
6 & 13 & 13 & 0 \\
7 & 11 & 10 & 1 \\
$8-14$ & 45 & 44 & 1 \\
$15-21$ & 17 & 17 & 0 \\
$21-28$ & 22 & 22 & 0 \\
\hline
\end{tabular}

"Time interval between maternal diagnosis of COVID infection and time till delivery and test of newborn

\section{Discussion}

Vertical transmission is the passage of a disease-causing agent (pathogen) from mother to baby during the period immediately before and/or after birth. ${ }^{8}$ Transmission might occur across the placenta, in the breast milk, or through direct contact during or after birth. Vertical transmission can occur through transplacental, transamniotic and ascending through vaginal contaminations. Perinatal transmission may occur due to close contact with mother and through milk. Definitions for vertical transmission of COVID-19 were given by Blumberg et al. ${ }^{9}$

Possibility of vertical transmission of SARS-CoV-2 has been proposed in previous studies; timing and rate of transmission need to be determined..$^{10}$ Information about diagnosis of vertical transmission was limited and also controversial views noted in the literature regarding the possibility of vertical transmission. According to a systematic review by Lamouroux, there was no evidence of vertical transmission. ${ }^{5}$ The relative risks and proportions of neonatal infection made by intrauterine transmission of SARS-CoV-2 from either transplacental or ascending infection, acquiring infection during labor and delivery, or following delivery from the mother, other individuals or the environment remain unknown. ${ }^{11}$

The first case of vertical transmission from mother to newborn was reported on March 26, 2020, in Wuhan China. ${ }^{10}$ They had detected IgM antibodies in the newborn within 2 hours 
of birth. Studies before these case reports did not find the proof of vertical transmission..$^{5,12,13}$ In systematic review by Yang in 33 studies involving 83 neonates delivered to SARS-CoV-2 infected mothers, 3 neonates were positive by RT-PCR at 16, 36, 72 hours of birth and another 6 had elevated virus-specific antibodies. Zeng et al. reported 3 out of 33 neonatal COVID infections due to possible vertical transmission. ${ }^{13}$ In a review by Pettirosso E of 60 articles, 19 neonates were reported to be SARS-CoV-2 positive after birth confirmed by reverse transcription polymerase chain reaction of nasopharyngeal swabs. ${ }^{14}$ In another review of 21 articles studying 86 neonates tested for vertical transmission by Ashraf et al., 4 newborns were positive for RT-PCR COVID, 3 were tested by nasopharyngeal swab, and 1 by amniotic fluid positive. ${ }^{15}$

In our study, SARS-CoV-2 infection was predominantly seen in 25-30 years age-group (40.2\%) and most were primipara (46.1\%); findings were similar to study by Nayak et al. ${ }^{16}$ Maternal COVID infection was diagnosed mainly by RT-PCR test as it was the only test available in India at the beginning of pandemic and IgM antibody and rapid antigen test were subsequently available and were utilized for maternal COVID-19 infection diagnosis. Majority of our patients were asymptomatic (93.1\%), and few had mild symptoms (6.1\%); these findings are in contrast with the review of SARS-CoV2-positive antennal women by Yang et al., where majority had fever $(87.5 \%)$ as presenting symptoms. ${ }^{3}$

Most of our patients (113 patients that is $56.2 \%$ ) delivered by caesarean section for obstetric indication, finding similar to other studies. ${ }^{2,16}$ A large number of patients were full term at the time of diagnosis of SARS-CoV-2, similar to study by Nayak et al. This may be due to initial government guidelines to screen only fullterm antenatal women for SARS-CoV-2 infection at beginning of the pandemic. Testing was also performed for all symptomatic antenatal women, irrespective of gestational age. RT-PCR of the nasopharyngeal swab of the newborn was utilized throughout the study for uniformity in protocol (Table 1). In our study, two newborns detected SARS-CoV-2 positive were delivered after 40 weeks of gestation. The rate of VT is $0.99 \%$ in our study. Preterm deliveries contributed to (12.9\%) of total deliveries, and all preterm newborns showed negative RT-PCR test for SARS-CoV-2. Our study had sufficiently large sample size as compared to previous studies in the literature. Similar study with small sample size also showed the positive newborn nasopharyngeal swabs taken within hours of birth. ${ }^{17,18}$ We have collected nasopharyngeal swabs immediately after birth or at least within 1 hour of birth so as to avoid falsepositive results by the colonization of virus from environmental (nosocomial source). RT-PCR is considered the gold standard for diagnosis of COVID-19 as per available literature.

To confirm the mechanism of vertical transmission by transplacental route will require immunohistochemistry of placenta for the confirmation of the presence of viral antigen by in situ hybridization. ${ }^{11}$ For hematogenous spread prolonged and intense viremia in maternal blood is must. One study showed $15 \%$ viremia in adults COVID patients. ${ }^{19}$

As per published data for SARS-CoV-2 infection to occur, ACE 2 receptor is essential to get infection to establish. As per Lamouroux, vertical transmission is not possible in the first trimester due to non-expression of angiotensin-converting enzyme receptor 2 (AEC2) expression in maternal-fetal interface. ${ }^{5}$ Between 6 and 16 weeks, ACE 2 receptor expression is detected. ${ }^{20}$ However, its expression is seen in later trimesters including in placenta and fetal organs. ${ }^{21}$ So, there is less possibility of vertical transmission in the first trimester than in the second and third trimester on the basis of ACE2 receptor expression. Expressed ACE2 receptors in maternal-fetal tissues may cause transmission, maternal viremia exposes virus to syncytiotrophoblast and cytotrophoblast receptors favoring transmission. Fetal capillary endothelium and erythroid cells ACE2 will need to be exposed to viruses to infect the fetus. This indicates that placenta, even if infected with SARS-CoV-2 in normal physiological state, effectively acts as a barrier for SARS-CoV-2. ${ }^{22}$ So, VT by hematogenous route is less likely to occur in early pregnancy where the natural fetoplacental barrier is fully functional.

Transamniotic mode is possible as COVID-19 is detected in amniotic fluid infecting mucous membrane of fetus. ${ }^{7}$ There may be possible transmission by ascending mode due to breach of natural placental barriers by infection or ruptured amniotic membrane exposing maternal vaginal secretions, which contain virus. $^{23}$

The SARS-CoV-2 genome is found in umbilical cord blood, the vaginal mucosa of a pregnant woman, and in at-term placenta. ${ }^{24}$ Evidence of SARS-CoV-2 RNA is found in milk specimens. ${ }^{7,24}$ Data indicate that in utero vertical transmission is possible in SARS-CoV2-positive pregnant women.

In addition, weeks of gestation may have an effect on vertical transmission. Our two cases of VT were delivered after crossing the expected date of delivery. There may be aging of natural placental barrier in postdated pregnancy or amniotic fluid leakage having the virus particle is a possible route of transmission, however needs detailed evaluation.

Both the mothers having newborns infected through VT did not have any high-risk conditions like preeclampsia, gestational diabetes, PROM, and chronic hypertension. So, these highrisk obstetrics conditions may not be influencing the vertical transmission.

Neonatal course was uneventful for non-COVID as well as COVID positive newborn in our study. Review by Di Mascio et al. found that early gestational age at infection, maternal ventilatory supports, and low birthweight are the main determinants of adverse perinatal outcomes in fetuses with maternal COVID-19. ${ }^{25,26}$

In our study, we have documented only one mode of vertical transmission that is intrauterine transmission; other modes of transmission like intrapartum and immediate postnatal transmission require repeated nasopharyngeal swab of newborns taken at regular interval after delivery. This study may initiate further hypothesis for other researchers to correlate vertical transmission with the severity of diseases and other modes of vertical transmission by larger sample size or carrying out multicenter study.

\section{Conclusion}

We conclude that vertical mode of transmission exists in SARSCoV-2 in pregnancy and vertical transmission rate of $0.99 \%$ is observed in our study. Vertical transmission was more common in postdated pregnancy; we could not find association with other obstetric high risks such as premature rupture of membrane with vertical transmission.

\section{Strength of Study}

Our study is carried out in the COVID dedicated hospital where facilities for COVID positive patients' delivery, NICU and ICU as well as laboratory facilities were available within campus 24 by 7 by qualified professionals. 


\section{Weakness of the Study}

This being hospital-based study, our results are based on the cases which are admitted to the hospital, while community-based study or multicenter study may give a clearer picture and wider inclusion of the data for the COVID-19 transmission during the delivery. The current study describes vertical transmission by intrauterine mode while vertical transmission through breastfeeding as well as close contact while breast-feeding may influence the vertical transmission rate, which was not a part of protocol in this study.

\section{Acknowledgments}

The authors would like to thank pathology and radiology department for providing necessary inputs of various data of COVID related cases. Our sincere gratitude goes to all supervisors, data collectors, ANMs, and study participants with their family members for their cooperation and support. The authors would like to thank our Dean Dr Rajendra Wable, and Board of Review Chairman Dr Mrs Ambike for granting necessary permissions and providing facilities for conducting study.

\section{Author's Contributions}

MA, HN, VY, SM, and TP were involved in all processes of this research work, including conception, design, supervision of data collection, data analysis, and write-up of the manuscript. All authors read and approved the final manuscript.

$M A$ and $H N$ conceived and designed the experiments. MA, HN, VY, SM, and TP performed the experiments. VY, HN, and MA analyzed the data. HN, VY, SM, and TP contributed materials/analysis tools. MA, HN, and VY wrote the paper.

\section{ORCID}

Hemraj Narkhede @i) https://orcid.org/0000-0001-7879-9420

\section{References}

1. Fan C, Lei D, Fang C, et al. Perinatal transmission of 2019 coronavirus disease-associated severe acute respiratory syndrome coronavirus 2: should we worry? Clin Infect Dis 2021;72(5):862-864. DOI: 10.1093/ $\mathrm{cid} / \mathrm{ciaa} 226$.

2. Della Gatta AN, Rizzo R, Pilu G, et al. Coronavirus disease 2019 during pregnancy: a systematic review of reported cases. Am J Obstet Gynecol 2020;223(1):36-41. DOI: 10.1016/j.ajog.2020.04.013.

3. Yang Z, Wang M, Zhu Z, et al. Coronavirus disease 2019 (COVID-19) and pregnancy: a systematic review. J Matern Fetal Neonatal Med 2020;1-4. DOI: 10.1080/14767058.2020.1759541 [Epub ahead of print].

4. Yang Z, Liu Y. Vertical transmission of severe acute respiratory syndrome coronavirus 2: a systematic review. Am J Perinatol 2020;37(10):1055-1060. DOI: 10.1055/s-0040-1712161.

5. Lamouroux A, Attie-Bitach T, Martinovic J, et al. Evidence for and against vertical transmission for severe acute respiratory syndrome coronavirus 2. Am J Obstet Gynecol 2020;223(1):91.e1-91.e4. DOI: 10.1016/j.ajog.2020.04.039.

6. Wang $\mathrm{C}$, Zhou $\mathrm{YH}$, Yang $\mathrm{HX}$, et al. Intrauterine vertical transmission of SARS-CoV-2: what we know so far. Ultrasound Obstet Gynecol 2020;55(6):724-725. DOI: 10.1002/uog.22045.

7. Vivanti AJ, Vauloup-Fellous C, Prevot S, et al. Transplacental transmission of SARS-CoV-2 infection. Nat Commun 2020;11:3572. DOI: $10.1038 / s 41467-020-17436-6$.

8. Fermin G. Chapter 5 - host range, host-virus interactions, and virus transmission. In: Tennant P, Fermin G, Foster JE, editors. Viruses. Academic Press; 2018. p. 101-134.
9. Blumberg DA, Underwood MA, Hedriana $\mathrm{HL}$, et al. Vertical transmission of SARS-CoV-2: what is the optimal definition? Am J Perinatol 2020;37(8):769-772. DOI: 10.1055/s-0040-1712457.

10. Dong L, Tian J, He S, et al. Possible vertical transmission of SARS-CoV-2 from an infected mother to her newborn. JAMA 2020;323(18):18461848. DOI: 10.1001/jama.2020.4621.

11. Schwartz DA, Morotti $D$, Beigi $B$, et al. Confirming vertical fetal infection with coronavirus disease 2019: neonatal and pathology criteria for early onset and transplacental transmission of severe acute respiratory syndrome coronavirus 2 from infected pregnant mothers. Arch Pathol Lab Med 2020;144(12):1451-1456. DOI: 10.5858/ arpa.2020-0442-SA.

12. Chen $\mathrm{H}$, Guo J, Wang C, et al. Clinical characteristics and intrauterine vertical transmission potential of COVID-19 infection in nine pregnant women: a retrospective review of medical records. Lancet (London, England) 2020;395(10226):809-815. DOI: 10.1016/S01406736(20)30360-3.

13. Zeng L, Xia S, Yuan $W$, et al. Neonatal early-onset infection with SARS-CoV-2 in 33 neonates born to mothers with COVID-19 in Wuhan, China. JAMA Pediatr 2020;174(7):722-725. DOI: 10.1001/ jamapediatrics.2020.0878.

14. Pettirosso E, Giles M, Cole S, et al. COVID-19 and pregnancy: a review of clinical characteristics, obstetric outcomes and vertical transmission. Aust N Z J Obstet Gynaecol 2020;60(5):640-659. DOI: 10.1111/ajo.13204.

15. Ashraf MA, Keshavarz P, Hosseinpour P, et al. Coronavirus disease 2019 (COVID-19): a systematic review of pregnancy and the possibility of vertical transmission. J Reprod Infertil 2020;21(3):157-168. PMCID: PMC7362089.

16. Nayak $\mathrm{AH}$, Kapote DS, Fonseca $\mathrm{M}$, et al. Impact of the coronavirus infection in pregnancy: a preliminary study of 141 patients. J Obstet Gynaecol India 2020;70(4):256-261. DOI: 10.1007/s13224-020-01335-3.

17. Schwartz DA, Mohagheghi P, Beigi B, et al. Spectrum of neonatal COVID-19 in Iran: 19 infants with SARS-CoV-2 perinatal infections with varying test results, clinical findings and outcomes. J Matern Fetal Neonatal Med 2020;1-10. DOI: 10.1080/14767058.2020. 1797672.

18. Patanè $L$, Morotti $D$, Giunta $M R$, et al. Vertical transmission of COVID-19: SARS-CoV-2 RNA on the fetal side of the placenta in pregnancies with COVID-19 positive mothers and neonates at birth. Am J Obstet Gynecol MFM 2020;2(3):100145. DOI: 10.1016/j. ajogmf.2020.100145.

19. Huang $C$, Wang $Y, L i X$, et al. Clinical features of patients infected with 2019 novel coronavirus in Wuhan, China. Lancet 2020;395(10223):497506. DOI: 10.1016/S0140-6736(20)30183 [Erratum in: Lancet 2020. PMID: 31986264; PMCID: PMC7159299].

20. Pringle KG, Tadros MA, Callister RJ, et al. The expression and localization of 74 the human placental prorenin/renin-angiotensin system throughout pregnancy: roles in 75 trophoblast invasion and angiogenesis? Placenta 2011;32(12):956-962. DOI: 10.1016/j. placenta.2011.09.020.

21. Li M, Chen L, Zhang J, et al. The SARS-CoV-2 receptor ACE2 expression of maternal-fetal interface and fetal organs by single-cell transcriptome study. PLoS One 2020;15(4):e0230295. DOI: 10.1371/ journal.pone.0230295.

22. Stagno S, Tinker MK, Elrod C, et al. Immunoglobulin M antibodies detected by enzyme-linked immunosorbent assay and radioimmunoassay in the diagnosis of cytomegalovirus infections in pregnant women and new-born infant. J Clin Microbiol 1985;21(6):930-935. DOI: 10.1128/jcm.21.6.930-935.1985.

23. Yu N, Li W, Kang Q, et al. Clinical features and obstetric and neonatal outcomes of pregnant patients with COVID-19 in Wuhan, China: a retrospective, single centre, descriptive study. Lancet Infect Dis 2020;20(5):559-564. DOI: 10.1016/S1473-3099(20)30176-6.

24. Fenizia C, Biasin M, Cetin I, et al. Analysis of SARS-CoV-2 vertical transmission during pregnancy. Nat Commun 2020;11(1):5128. DOI: 10.1038/s41467-020-18933-4. 
25. Yang $\mathrm{R}$, Mei $\mathrm{H}$, Zheng T, et al. Pregnant women with COVID-19 and risk of adverse birth outcomes and maternal-fetal vertical transmission: a population-based cohort study in Wuhan, China. BMC Med 2020;18(1):330. DOI: 10.1186/s12916-020-01798-1.
26. Di Mascio D, Sen C, Saccone G, et al. Risk factors associated with adverse fetal outcomes in pregnancies affected by Coronavirus disease 2019 (COVID-19): a secondary analysis of the WAPM study on COVID-19. J Perinat Med 2020;49(1):111-115. DOI: 10.1515/jpm-2020-0539. 